\title{
Glucosamine hydrochloride for the treatment of osteoarthritis symptoms
}

\author{
Beth Anne Fox \\ Mary M Stephens \\ East Tennessee State University, \\ Family Physicians of Kingsport, \\ Kingsport, TN, USA
}

Correspondence: Beth Anne Fox East Tennessee State University, Family Physicians of Kingsport, 20I Cassel Drive, Kingsport, Tennessee 37660, USA

Tel + I 4232459623

Fax +I 423245963

Email foxba@etsu.edu

\begin{abstract}
Osteoarthritis is the most common arthritis in the world. It affects millions of people with age being the greatest risk factor for developing the disease. The burden of disease will worsen with the aging of the world's population. The disease causes pain and functional disability. The direct costs of osteoarthritis include hospital and physician visits, medications, and assistive services. The indirect costs include work absences and lost wages. Many studies have sought to find a therapy to relieve pain and reduce disability. Glucosamine hydrochloride $(\mathrm{HCl})$ is one of these therapies. There are limited studies of glucosamine $\mathrm{HCl}$ in humans. Although some subjects do report statistically significant improvement in pain and function from products combining glucosamine $\mathrm{HCl}$ and other agents, glucosamine $\mathrm{HCl}$ by itself appears to offer little benefit to those suffering from osteoarthritis.
\end{abstract}

Keywords: osteoarthritis, glucosamine $\mathrm{HCl}$, middle aged, aged, humans

Osteoarthritis (OA) is the most common arthritis in the world. It is characterized by degeneration of joint cartilage which can result in bony remodeling and synovitis. The diagnosis is primarily a clinical one based on history and physical examination findings. The need for radiographic findings to confirm the diagnosis is controversial since the degree of pain does not correlate to the severity of disease radiographically (Hannan et al 2000). The hip and knee joints are most commonly involved; however, the shoulders, hands, and back are also frequently affected. Those afflicted with this condition present with pain, morning stiffness of less than an hour with progressive improvement during the day, and disability due to loss of function and with an inability to perform routine daily activities.

\section{Burden of suffering}

In 2000, an estimated $9.6 \%$ of men and $18 \%$ of women over 60 had symptomatic OA (Woolf and Pfleger 2003). With age being the greatest risk factor for acquiring OA, the burden of disease can only worsen. By 2050, the over-60 population in Europe is estimated to reach about $33 \%$ of the population and in North America about $27 \%$ of the population. The greatest growth will be seen in the octogenarians and above with a greater than 100\% increase anticipated by 2050 (Ethgen and Reginster 2004). It is not surprising then, that $\mathrm{OA}$ was deemed to be a significant enough problem worldwide to prompt the World Health Organization (WHO) to designate 2000-2010 as the Bone and Joint Decade (Woolf and Pfleger 2003).

Not only is the burden of disease exemplified by the sheer numbers of people with the disease but also by the frequency of individual suffering. About $25 \%$ of persons over 55 experience knee pain most days of the month (Felson 2006). Utilizing joint pain as a marker of arthritis, $11 \%$ of Oxfordshire residents over 65 years of age surveyed 
reported hip and knee pain and $41 \%$ had hip or knee pain for at least a month (Dawson et al 2004).

\section{Risk factors}

There are some modifiable and nonmodifiable risk factors for OA. Under the age of 45 the risk of OA is more common in men. The risk rises and is more common in women after 55 years of age. It is more likely to affect those with obesity; those with advancing age; and those with certain lifestyle issues such as sports interests like running and prior joint injuries; those with particular employment histories especially farming; and in some with genetic predisposition. OA of the hand may be more associated with inheritance than other joint presentations.

OA patterns vary along ethnic and cultural lines. Blacks from Jamaica, South Africa, Nigeria, and Liberia have lower hip OA prevalence compared with Europeans, as does the Asian population. OA can manifest in a single joint. However, when multiple joints are affected, everyday activities such as ambulation become more difficult to perform as a result of the pain, swelling, and stiffness associated with the disease resulting in poorer quality of life outcomes (Keenan et al 2006). Chronic diseases in general result in impaired functioning across various physical, mental, and psychosocial parameters. Musculoskeletal conditions have been linked to poorer quality of life parameters than cardiovascular, neurologic, endocrine, and renal diseases primarily as a result of physical impairment, disability, and poorer life satisfaction (Sprangers et al 2000).

Osteoarthritis not only affects the individual but has a significant impact on healthcare costs, employer costs, and job performance. These are divided into direct and indirect costs. Direct costs attributable to arthritis include hospital and outpatient visits, pharmaceuticals, home health services and assistive devices. In 75-79 year olds with OA, prescription drug costs were $102 \%$ higher than a similar cohort without the disease and their outpatient visits were more that doubled (Mapel et al 2004). Similar high costs were noted in this earlier trial for persons with a diagnosis of OA with increases seen in the areas of diagnostic testing, physician visits, and other direct medical costs. Prescriptions were substantially more significant in those with OA than in those without this diagnosis (Gabriel et al 1997). Indirect costs include lost wages and work absences. During a two week period, workers ages 40-65 who experienced exacerbation of pain related to OA had more lost wages and time away from work than workers without exacerbations. These costs were estimated at over $\$ 7$ billion per year. About $66 \%$ of this amount was attributed to only $38 \%$ of the surveyed workforce (Ricci et al 2005).

\section{Glucosamine hydrochloride}

One complementary medication that has been investigated for the treatment of OA is glucosamine. Glucosamine is a derivative of cellular glucose metabolism. It is also a component of glycosaminoglycans and proteoglycans of the cartilage matrix covering the ends of bones and hyaluronic acid which is a part of synovial fluid within the joint. The primary source of exogenous glucosamine is the exoskeleton of shellfish and exists in primarily two formulations, glucosamine hydrochloride $(\mathrm{HCl})$ and glucosamine sulfate (Institute of Medicine and National Research Council 2005). Glucosamine sulfate requires compound stabilizers in the form of salts and has $74 \%$ purity. Glucosamine $\mathrm{HCl}$ lacks the sulfate group and has $99 \%$ purity. Therefore, glucosamine $\mathrm{HCl}$ in a dosage of $1,500 \mathrm{mg}$ equals a dosage of 2,608 $\mathrm{mg}$ of glucosamine sulfate (Owens et al 2004). Glucosamine is readily absorbed from the gastrointestinal tract with oral administration, rapidly undergoes metabolism via the liver and the first pass effect, and is eliminated through the feces and urine. Peak levels are in about 8 hours after oral ingestion primarily due to about 90\% protein binding (Anderson et al 2005).

The mechanism of action of glucosamine in humans is unknown. Because glucosamine is a part of the cartilage matrix in joint tissues, it has been theorized for many years that its administration could effect symptomatic relief for OA sufferers by supplying the components for cartilage repair and thus improve pain and disability. More recently, however, it has been demonstrated in animal models that glucosamine has an anti-inflammatory effect via the reduction of nuclear factor kappa beta induced by interleukin-1 (IL-1) (Gouze et al 2002, 2006). A few studies in humans have revealed that glucosamine $\mathrm{HCl}$ reduces IL-1 stimulated production of catabolic enzymes and inflammatory markers such as prostaglandin E2 by chondrocyte and synovial cells harvested from surgical specimens removed from patients with OA (Nakamura et al 2004; Uitterlinden et al 2006).

\section{Literature review}

For this review, the focus has been limited to the use of glucosamine $\mathrm{HCl}$ preparations for symptomatic relief of OA in humans. A Medline search was undertaken utilizing the search terms "glucosamine hydrochloride" and "osteoarthritis". Secondary search terms were "human trials" and "ages 45 and older". Studies were found that specified the use of glucosamine $\mathrm{HCl}$ in humans were included. Other 
studies were reviewed and discarded because they focused on alternate preparations of glucosamine.

The most recent and largest randomized controlled trial (RCT) was a multi-center trial supported by the National Institute of Health, called the Glucosamine/chondroitin Arthritis Intervention Trial (GAIT). This study sought to evaluate glucosamine $\mathrm{HCl}$, chondroitin sulfate, glucosamine $\mathrm{HCl}$ combined with chondroitin sulfate, celecoxib, or placebo for the pain associated with knee OA for a duration of 24 weeks. This study enrolled 1583 patients. Enrollees had to be at least 40 years of age, have suffered with knee pain for at least 6 months, and have radiographic evidence of OA. The five regimens studied were: 1) Glucosamine $\mathrm{HCl} 500 \mathrm{mg}$ three times a day, 2) Chondroitin sulfate $400 \mathrm{mg}$ three times a day, 3) Glucosamine $\mathrm{HCl} 500 \mathrm{mg}$ plus chondroitin sulfate $400 \mathrm{mg}$ three times a day, 4) celecoxib $200 \mathrm{mg}$ daily, or 5) placebo. Each of the five groups had over 300 persons assigned and had a mean age of 59 years and a mean body mass index of 32. Most of the participants, $64 \%$, were women. Participants could take up to $4000 \mathrm{mg}$ of acetaminophen as rescue medication per day, excluding the 24 hour period prior to clinical evaluation at $4,8,16$, and 24 weeks.

The primary outcome measurement was a $20 \%$ decrease in the Western Ontario and McMaster Osteoarthritis (WOMAC), score from baseline to week 24 , but secondary assessments included evaluation by the patient and evaluator of disease state and therapy response, changes in pain scores using the visual analog scale (VAS), quality of life assessed by the SF46 General Health Survey, and rescue medication use. The WOMAC Index is a 24 question validated survey assessing pain, function, and mobility. The VAS is a self-assessment of pain and function. Unlike other studies, the compounds used in this study were analyzed and certified by the Food and Drug Administration. There was a withdrawal rate of about $20 \%$ among all groups.

Overall, there was no difference among the treatment groups compared with placebo in a combined analysis for the primary outcome measure. Sub-group analysis was also performed. In the $78 \%$ of those with mild OA, there was no significant change when compared with placebo for the 4 therapy groups. Treatment effects ranged from a low of $63 \%$ response rate in the glucosamine $\mathrm{HCl}$ and chondroitin combination group to a high of $70 \%$ in the celecoxib group. None of these approached significance however. In the moderate-tosevere OA group ( $22 \%$ of participants), there was improvement in the primary outcome measure, the WOMAC score, in the glucosamine $\mathrm{HCl}$ plus chondroitin sulfate treatment group at a $\mathrm{P}$ value of 0.002 when compared with placebo but there was no significant improvement noted in the other therapy groups. Acetaminophen use was low among all groups. All groups had gradual improvement in pain over the 24 weeks but the celecoxib group had the fastest improvement at 4 weeks. The numbers of participants in the moderate-to-severe OA group was small (Clegg et al 2006).

An earlier study included 50 volunteers, ages 20 to 70 years of age, experiencing knee pain and functional disability and evaluated glucosamine $\mathrm{HCl}$ in dosages of 2,000 mg per day compared with placebo over a 90 day period. Knee pain was assessed using The Knee Pain Scale and the Knee Injury and Osteoarthritis Outcome Score which have been designed to quantify knee pain and mobility. A complete history was taken from each participant regarding knee pain along with any prior evaluations and treatments received that might suggest cartilage degeneration. There were 46 subjects completing the study, 24 in the glucosamine group and 22 in the placebo group. The participants underwent four assessment sessions and each time completed the two questionnaires, underwent joint line palpation, performed a "duck walk", and climbed 32 stairs for 5 rotations. Of note, there were 15 people in the placebo arm that had greater than 10 years of symptoms and only 7 in the glucosamine arm that had symptoms for more that 10 years. Otherwise the two groups were fairly evenly divided along gender and age lines. Prescription and anti-inflammatory medications were allowed and recorded by the participants. Only 4 participants used anti-inflammatory medications, one on a regular basis and 3 as rescue medications only. At week eight, those receiving glucosamine $\mathrm{HCl}$ had significant improvement in pain and mobility $(\mathrm{P}=0.004)$. By week twelve, $88 \%$ felt their pain had improved compared with only $17 \%$ of the placebo group. No significant improvement between the groups was demonstrated with joint line palpation, "duck walk", or stairclimbing. Although subjective improvement was noted in this study, its significance is questionable due to the small number of participants (Braham et al 2003).

Another study utilizing glucosamine $\mathrm{HCl}$ focused on patients with OA of the knee. The primary end point was a change in the WOMAC Index. A Likert Pain Scale was also administered to participants. Volunteers for the study were solicited via newspaper advertisements with 1100 responding and 118 meeting criteria for participation. Participants had to describe at least moderate knee pain for 6 months and have radiographic changes consistent with OA. They could not have used glucosamine previously or oral steroids. Subjects were given glucosamine $\mathrm{HCl} 500 \mathrm{mg}$ to take three times a day which was provided by the manufacturer. Rescue medication 
was also permitted in the form of acetaminophen $500 \mathrm{mg}$ that could be taken to a maximum of $4000 \mathrm{mg}$ per day. One hundred one subjects completed the 8 week trial. Although improvements were seen in the glucosamine group in 23 of the 24 questions of the WOMAC, this was not statistically significant from the placebo group. Improvements were suggested however during the knee examination and documented in daily diaries by those given glucosamine. No differences were noted between the groups in acetaminophen use or side effect profiles (Houpt et al 1999).

An internet-based study was conducted by McAlindon and colleagues (2004) to evaluate the effectiveness of glucosamine for control of symptoms resulting from knee OA. The solicitation, interview, and selection of participants were entirely conducted over the internet. Participants had to be at least 45 years of age and have radiographic documentation consistent with joint space loss. Exclusion criteria included those using glucosamine, having a knee injection within 60 days, history of arthroplasty, or allergy to shellfish. Initially the glucosamine utilized was a sulfate preparation. However during the trial, the manufacturer withdrew the supply and the preparation was changed to glucosamine $\mathrm{HCl}$ obtained from another pharmaceutical company. Two hundred five participants were divided between the glucosamine and the placebo groups. There was an overall reduction in pain scores during the study among both groups but no significant difference between the groups. Notably, there was a larger change in pain scored with the glucosamine $\mathrm{HCl}$ group than the glucosamine sulfate group but neither of the preparations significantly improved pain when compared with placebo. Even though assignment to groups was not standardized for gender, weight, or nonsteroidal antiinflammatory drug (NSAID) use; the placebo group had more women, heavier participants as indicated by a higher body mass index, and used more NSAIDs; there remained no difference among the study groups when adjustments were made for these variables. Two important aspects of this study that may have affected the outcome have to do with it being solely internet based and utilization of two different compounds of glucosamine (McAlindon et al 2004).

Another study sought to determine the effectiveness of glucosamine in the reduction of pain in OA of the knee. Participants had to have a history of OA and radiographic findings consistent with the disease. Ninety eight people ages 34 to 81 were evenly assigned to the glucosamine and the placebo groups. Therapy consisted of glucosamine (preparation not specified) $500 \mathrm{mg}$ three times a day for 60 days. Other analgesics could be continued by participants as needed.
Pain was assessed using the VAS while at rest and then while walking. There was no significant difference between the mean scores of pain intensity between the two groups at rest or walking at 30 or 60 days. Glucosamine was found to be no more effective than placebo for relieving knee pain in this RCT (Rindone et al 2000).

Two RCTs evaluated the effect of a combination product in degenerative joint disease (DJD) and OA of the knee and one also included DJD of the back. This combination included $1500 \mathrm{mg}$ of glucosamine $\mathrm{HCl}, 1200 \mathrm{mg}$ of chondroitin sulfate, and $228 \mathrm{mg}$ of manganese ascorbate. In one of these RCTs, 34 male military personnel with knee or low back pain for 3 months and radiographic evidence of OA were included. Of the 21 knee participants, 20 met criteria for OA. This placebo-controlled crossover study was for a period of 16 weeks with only acetaminophen permitted as additional medication for pain. There was significant improvement in knee symptoms. This was demonstrated by improvement in pain assessed by the VAS recorded at clinic visits and recorded in diaries, personal assessment of treatment, and overall physical examination scores, but not the individual parameters of tenderness, effusion, swelling, and warmth. There was no significant change noted between the medication and placebo arms in any of the other measured parameters of acetaminophen use, running, stair-climbing, and range of motion (Leffler et al 1999).

In the other RCT, the same combination of nutraceuticals was used for the treatment of participants with knee OA, but the dosage was $2000 \mathrm{mg}$ of glucosamine $\mathrm{HCl}, 1600 \mathrm{mg}$ of chondroitin sulfate, and $304 \mathrm{mg}$ of manganese ascorbate per day in divided doses. Participants were followed for 6 months and evaluated every two months using the validated Lequesne questionnaire of disease severity, with a $25 \%$ decrease in this measurement as the primary outcome. Secondary measures were the WOMAC questionnaire and the patient's own global assessment of their OA using the visual analog scale. Rescue medications allowed included NSAIDs and acetaminophen. Eligible participants were 45-75 years of age and 93 were accepted for inclusion. There were 46 in the treatment arm and 47 received placebos. Mild or moderate $\mathrm{OA}$ was present in 33 of those receiving medication and 39 receiving placebo and severe $\mathrm{OA}$ in 13 and 8 in treatment and placebo arms respectively. There was improvement noted in those with mild and moderate $\mathrm{OA}$ with treatment intervention at $52 \%$ compared with $28 \%$ with placebo at four and six months only. There was no difference with intervention or placebo in those with severe knee OA, either group when assessed with the WOMAC questionnaire for assessment of 
pain, function, and joint stiffness, or patients' assessments of response. The reduction in acetaminophen use from 2 to 6 months approached but did not reach significance. Of note, this study was sponsored in part by the manufacturer of the glucosamine, chondroitin, and manganese ascorbate product (Das and Hammad 2000).

\section{Adverse events}

Side-effects and adverse events related to glucosamine $\mathrm{HCl}$ were mild and fairly consistent across all studies. Primarily side-effects were related to the gastrointestinal tract and included dyspepsia, nausea, vomiting, diarrhea, and constipation. Other effects include headache, bad taste, and fatigue. More serious events reported included the onset of diabetes, hypothyroidism, peripheral edema, sexual dysfunction, elevated blood pressure, and chest pain. Most side effects did not require discontinuation of therapy or withdrawal from the study; however, a few were unable to tolerate the symptoms and did withdraw (Houpt et al 1999; Leffler et al 1999; Das and Hammad 2000; Rindone et al 2000; McAlindon et al 2004; Clegg et al 2006). There are no long term studies of side-effects, adverse events, or toxicity of glucosamine $\mathrm{HCl}$.

\section{Conclusion}

Osteoarthritis, particularly of the hip and knee, can severely affect the health and well-being of those who suffer with the disease. The population of the world is aging and so is the burden of disease of OA. In those afflicted with the disease, a majority of the disease burden will be in those over 65 years of age. A substantial amount of the healthcare dollars spent for OA involves direct costs that are associated with outpatient evaluation, treatment modalities especially medications, and assistive services and equipment. Indirect costs can also be enormous when including lost time from work and associated lost wages.

Not only because of the economic burden of OA but also because of the poor health outcomes and quality of life experienced by those with the disease and with advancing symptoms with age, therapies to reduce symptoms especially pain exacerbations, improve quality of life, and perhaps slow the progression of the disease are being explored. Other benefits would be if the therapy had few side effects, were minor in nature, and was reasonably priced and readily available. One such potential modality is glucosamine. Initial studies for this therapy were done in Europe more than 30 years ago and were sponsored by the glucosamine manufacturers. The studies lacked standardized randomization, standardization of medication compounding, and suffered from poor masking of participants and most did not adhere to the intention-to-treat analysis of data. However, many of these initial studies demonstrated improvement in pain and function in the glucosamine therapy groups when compared with placebo or at times to a particular NSAID.

Because of these initial positive outcomes, additional studies have been undertaken to remove some of the initial biases and to attempt to duplicate the results. There is little data on glucosamine $\mathrm{HCl}$ since most studies have utilized glucosamine sulfate and not glucosamine $\mathrm{HCl}$. Only one of the RCTs reviewed in this article demonstrated any significant improvement in outcomes with glucosamine $\mathrm{HCl}$ alone over placebo or NSAID. Some studies did suggest improvement but the results were not statistically significant.

In the largest, most talked about and awaited trial, the GAIT trial, the results were confusing and difficult to interpret. Statistically significant improvement was seen only in those with moderate-to-severe knee OA with the combination therapy of glucosamine $\mathrm{HCl}$ and chondroitin sulfate; however, only small numbers of subjects were involved. Another combination product of glucosamine $\mathrm{HCl}$, chondroitin sulfate, and manganese ascorbate was compared with placebo in two earlier RCTs and improvement in pain and function were seen in those with mild-to-moderate OA.

In conclusion, this current review of the available literature suggests little benefit with the use of glucosamine $\mathrm{HCl}$ alone to those suffering from OA. Certain combination products may be of benefit in selected subgroups of patients.

\section{Acknowledgments}

The authors would like to thank Ivy Click, M.A. for her assistance in the literature search and assistance in preparation of the manuscript for submission.

\section{References}

Anderson JW, Nicolosi RJ, Borzelleca JF. 2005. Glucosamine effects in humans: a review of effects on glucose metabolism, side effects, safety considerations and efficacy. Food Chem Toxicol, 43:187-201.

Braham R, Dawson B, Goodman C. 2003. The effect of glucosamine supplementation on people experiencing regular knee pain. Br J Sports Med, 37:45-9; discussion 9.

Clegg DO, Reda DJ, Harris CL, et al. 2006. Glucosamine, chondroitin sulfate, and the two in combination for painful knee osteoarthritis. N Engl J Med, 354:795-808.

Das A Jr, Hammad TA. 2000. Efficacy of a combination of FCHG49 glucosamine hydrochloride, TRH122 low molecular weight sodium chondroitin sulfate and manganese ascorbate in the management of knee osteoarthritis. Osteoarthritis Cartilage, 8:343-50.

Dawson J, Linsell L, Zondervan K, et al. 2004. Epidemiology of hip and knee pain and its impact on overall health status in older adults. Rheumatology (Oxford), 43:497-504. 
Ethgen O, Reginster JY. 2004. Degenerative musculoskeletal disease. Ann Rheum Dis, 63:1-3.

Felson DT. 2006. Clinical practice. Osteoarthritis of the knee. $N$ Engl $J$ Med, 354:841-8.

Gabriel SE, Crowson CS, Campion ME, et al. 1997. Direct medical costs unique to people with arthritis. J Rheumatol, 24:719-25.

Gouze JN, Bianchi A, Becuwe P, et al. 2002. Glucosamine modulates IL-1-induced activation of rat chondrocytes at a receptor level, and by inhibiting the NF-кB pathway. FEBS Lett, 510:166-70.

Gouze JN, Gouze E, Popp MP, et al. 2006. Exogenous glucosamine globally protects chodrocytes from the arthritogenic effects of IL-1 $\beta$. Arthritis Res Ther, 8:R173.

Hannan MT, Felson DT, Pincus T. 2000. Analysis of the discordance between radiographic changes and knee pain in osteoarthritis of the knee. $J$ Rheumatol, 27:1513-7.

Houpt JB, McMillan R, Wein C, et al. 1999. Effect of glucosamine hydrochloride in the treatment of pain of osteoarthritis of the knee. $J$ Rheumatol, 26:2423-30.

Institute of Medicine, National Research Council. 2005. Glucosamine: Prototype monograph summary. In Dietary Supplements: A Framework for Evaluating Safety. Washington, DC: The National Academies. p 363-6.

Keenan AM, Tennant A, Fear J, et al. 2006. Impact of multiple joint problems on daily living tasks in people in the community over age fifty-five. Arthritis Rheum, 55:757-64.

Leffler CT, Philippi AF, Leffler SG, et al. 1999. Glucosamine, chondroitin, and manganese ascorbate for degenerative joint disease of the knee or low back: a randomized, double-blind, placebo-controlled pilot study. Mil Med, 164:85-91.
Mapel DW, Shainline M, Paez K, et al. 2004. Hospital, pharmacy, and outpatient costs for osteoarthritis and chronic back pain. J Rheumatol, 31:573-83.

McAlindon T, Formica M, LaValley M, et al. 2004. Effectiveness of glucosamine for symptoms of knee osteoarthritis: results from an internet-based randomized double-blind controlled trial. Am J Med, 117:643-9.

Nakamura H, Shibakawa A, Tanaka M, et al. 2004. Effects of glucosamine hydrochloride on the production of prostaglandin E2, nitric oxide and metalloproteases by chondrocytes and synoviocytes in osteoarthritis. Clin Exp Rheumatol, 22:293-9.

Owens S, Wagner P, Vangsness CT Jr. 2004. Recent advances in glucosamine and chondroitin supplementation. J Knee Surg, 17:185-93.

Ricci JA, Stewart WF, Chee E, et al. 2005. Pain exacerbation as a major source of lost productive time in US workers with arthritis. Arthritis Rheum, 53:673-81.

Rindone JP, Hiller D, Collacott E, et al. 2000. Randomized, controlled trial of glucosamine for treating osteoarthritis of the knee. West $J$ Med, 172:91-4.

Sprangers MA, de Regt EB, Andries F, et al. 2000. Which chronic conditions are associated with better or poorer quality of life? J Clin Epidemiol, 53:895-907.

Uitterlinden EJ, Jahr H, Koevoet JL, et al. 2006. Glucosamine decreases expression of anabolic and catabolic genes in human osteoarthritic cartilage explants. Osteoarthritis Cartilage, 14:250-7.

Woolf AD, Pfleger B. 2003. Burden of major musculoskeletal conditions. Bull World Health Organ, 81:646-56. 